\title{
What can the Maker Movement Teach Us about the Digitization of Creativity?
}

In recent years, the 'maker movement' has emerged as a social phenomenon driven by novel technological possibilities. With the help of inexpensive, yet highly versatile means of production (e.g., CNC milling machines, 3D printers) and easy-to-use software tools, makers free themselves from their traditional role as passive consumers and evolve into innovators and producers. Although the act of physical production seems to be at the centre of the movement, a large part of the creative work takes place in the online sphere. These digital activities and their outcomes provide a rich source of information that can be used to gain a more nuanced understanding of how the digitization affects the creative process itself.

Of all the production methods available to makers, 3D printing is probably the most versatile and requires only a limited understanding of the production process. Several 3D design software packages allow even lay people to turn their ideas into printable designs. This combination of flexibility and usability has led to an abundance of 3D object models over the past years, which are shared and jointly refined with the community on digital maker platforms. As part of a multi-year research project on the use of 3D printing by the maker community, we found that the use of these platforms in the creative process blurs the boundaries between the digital and the physical and ultimately changes the way ideas are expressed, curated, and eventually translated into physical reality. In particular, we saw how makers with entirely different backgrounds (e.g., HW/SW developers, designers, business and social entrepreneurs) traverse across the startup world, software development, and open online communities, to combine concepts through a novel digitized creative process.

When interviewing makers on why they started particular projects we found that their creative processes were initiated by one of two triggers: a problem trigger or a curiosity trigger. In the first case, creative efforts are made to solve a particular problem, whereas in the latter case, curiosity about the technology and enjoyment of the creative act itself are motivating factors. Traditional creativity methods like 'design thinking' emphasize that a creative process needs to start with an in-depth understanding of a given problem [2]. Among makers we see that this precondition is not imperative as they iteratively improve their problem-solution-fit along the way.[3]

Regardless of the trigger, the subsequent creative process can be divided into three phases: an inspiration phase, a distribution phase, and an iteration phase.

\section{Inspiration Phase: Where Ideas Come From}

Creativity in a digital context often makes use of prior art. It embodies the past in the present and is therefore a reflection of the social context in which it takes place. It is inherently social when makers of an online community build upon each other's work through 'remixing', a process that resembles versioning and code sharing in software repositories. An example of this can be found on Thingiverse, the world's largest 3D printing community. Here, designs are shared under open licenses (e.g., CC BY) that explicitly allow remixing. We spoke to many 
designers who browse the platform in search for inspiration. And once they find an inspiring design they employ remixing to turn it into something new.

We found that the creative processes behind these remixes are not as chaotic as one might expect, they follow distinct patterns. Remixing is either additive, that is, multiple ideas are combined into something new, or subtractive, that is, something is omitted to focus on key elements. An example of an additive remix is the debate coin in Figure 1 (A), maker Karr placed the mascots of the US's Republican and Democratic Party on a printable coin. By tossing the coin, a user can decide between the parties.

Figure 1: On the left a debate coin[4] that is remixed from two political mascots ${ }_{[5] 6]}$, on the right plant signs ${ }^{[]]}$that were remixed into bookmarks ${ }^{[8]}$

Remixing is also used to bring together knowledge from separate domains. On Thingiverse, designs are grouped into categories like 'Household' or 'Learning'. In many cases makers transfer ideas from one category to another where these ideas are not yet known. An example can be found in Figure 1 (B). Maker skarab found plant signs that allow gardeners to remember which pot contains which plant. He transferred the idea from 'Outdoor \& Garden' to the 'Office' category by turning the signs into bookmarks that can be clipped to magazines, documents, or books. Instead of plant names, the bookmarks provide prompts like 'to do', 'please sign', or 'read this'.

Introducing new aspects to a field is a balancing act. If makers introduce too much newness, their designs might be hard to understand and ultimately fail. If they introduce too few novel aspects their designs are considered "nothing new" and fail as well. A similar situation is well documented in research on scientific impact where "science follows a nearly universal pattern: The highest-impact science is primarily grounded in exceptionally conventional combinations of prior work yet simultaneously features an intrusion of unusual combinations." $[9]$

\section{Distribution Phase: Reaching Users Early}

It is a notorious problem in new product development that many new ideas fail. In some industries, like fast-moving consumer goods, failure rates are typically above 50\%.10] The maker community is not much different, many designs fail to reach a large user base and receive little feedback. However, we saw that makers adapt to this, they understand that other people's future needs are hard to predict and in order to make meaningful contributions, they actively seek community feedback as early as possible.

In the analog age, one of the biggest obstacles for many creatives was to find an effective means for reaching potential users. Distribution was expensive and controlled by a few gatekeepers (e.g., publishers or record companies). And once a product was in distribution, it was impractical to change it especially with large-scale production. However, in the maker community, production and distribution often go hand in hand. Online platforms such as Shapeways or MyMiniFactory make distribution easy for small-scale production. And the 
platforms typically provide the producers with a medium for receiving feedback. This is in stark contrast to traditional creative processes where only a finished solution is distributed. The digitization of creativity does not end with distribution. Rather, online platforms allow makers to distribute early in the process, which in turn allows them to iteratively improve their problemsolution-fit.

Maker Jonathan Bobrow provides an example (Figure 2). In 2013, Apple changed the charging ports on their laptops. To use an old charger a tiny adapter was needed, Bobrow lost his frequently. To solve this problem, he developed a key ring to hold the adapter. Assuming that others had the same problem, he offered his key ring on the 3D printing marketplace Shapeways. As the product took off, Bobrow founded a company, improved the design and launched a campaign on the crowdfunding platform Kickstarter111. Hundreds of Mac users supported the campaign and made 'keybit' a success.

Figure 2: Jonathan Bodrow's keybit: From a sketch to a product

Going to users early to collect initial feedback resembles the so-called 'Lean Startup' concept from the startup world. ${ }^{12]}$ Here, companies enter a market as early as possible to test their business models. They pay close attention to feedback and orientate their business to what they hear. This strategy allows them to prioritize development efforts more effectively, as the feedback they receive gives them suggestions on what to do next. All across the maker community we found similar behaviors: makers offering solutions free of charge act this way, but so do hardware developers. New printers or tools are typically introduced via crowdfunding platforms like Kickstarter or Indiegogo. Here, developers can judge the market's reaction to their solution. Successful ideas can receive full attention, while those that don't attract audiences can offer learning opportunities before significant production costs accrue.

\section{Iteration Phase: Steadily Improving Solutions}

In the third phase of the creative process, makers iteratively improve their concepts. To do so they rely on intensive interactions with users. These interactions serve two purposes.

Firstly, they increase the fit between ideas and user needs. In this regard, makers update existing designs to improve the problem-solution-fit. This behavior is in line with other creative communities and well documented in the field of innovation management where it is often referred to as 'user integration'. In one case maker AdamStag developed 18 versions of a single bird whistle, improving the product and answering user comments. Users for instance asked for water level marks to make the whistle more intuitive, a feature AdamStag promptly implemented (Figure 3).

Secondly, makers benefit from user feedback to speed up their creative activities. Getting feedback early makes their creative processes more focused and at the same time more flexible. This refocusing during the development process resembles concepts from software development like 'agile'[13]. 
Figure 3: Feedback and new iterations of a bird whistle on the 3D printing website Thingiverse ${ }^{[14]}$

\section{Conclusion}

In a world where the creative process does not end with the distribution of a product, we need to rethink our understanding of how people create. The maker community offers a great learning opportunity for all of us as they openly experiment with the creative process. But others do too: rap musician Kanye West, for instance, updated his album 'The Life of Pablo' after its official release date, describing this as a "living breathing changing creative expression." $[15]$ Another pop cultural example is the original Star Wars trilogy, which was reedited several times after its initial release, but in this case much to the dismay of the movies' fanbase. In the future, we will see an increasing digitization of creative processes as more and more products bridge the digital and the physical world. The development in the maker movement is only one manifestation of this. Against this background, it is helpful to investigate how new technologies combined with concepts from software development and the startup world can help create a digitized creative process (an overview can be found in Figure 4). With the 'digitization of the physical' our creative processes become more fluid and, in turn, even physical goods become inspired by processes from the world of bits. The digitization of the creative process not only brings about frequent interaction with fellow designers but also provides users with the earlier versions of a prototype in an incremental and iterative manner, which over time leads to a better problem-solution-fit. Restructuring existing creative processes along these learnings will help us to keep them up to date and question decade old assumptions on how to shape and control creativity.

Figure 4: Overview of the digitized creative process

\footnotetext{
[1] ANDERSON, C. 2012. Makers. The New Industrial Revolution, New York, Crown Business.

[2] https://www.interaction-design.org/literature/article/5-stages-in-the-design-thinking-process

[3] Von Hippel, E., \& Von Krogh, G. (2015). Crossroads-Identifying viable "need-solution pairs": Problem solving without problem formulation. Organization Science, 27(1), 207-221.

[4] http://www.thingiverse.com/thing:495777

[5] http://www.thingiverse.com/thing:32971

[6] http://www.thingiverse.com/thing:32970

[7] http://www.thingiverse.com/thing:1013494

[8] http://www.thingiverse.com/thing:1039106

[9] Uzzi, B., Mukherjee, S., Stringer, M., \& Jones, B. (2013). Atypical combinations and scientific impact. Science, 342(6157), 468-472. QUOTE ON PAGE 468

[10] Schneider, J., Hall, J. (2011). Why Most Product Launches Fail. Harvard Business Review, April 2011.

[11] https://www.kickstarter.com/projects/jbobrow/keybit-magsafe-adapter-key-ring

[12] Ries, E. (2011). The lean startup: How today's entrepreneurs use continuous innovation to create radically successful businesses. Crown Books.

[13] Beck, K., Beedle, M., Van Bennekum, A., Cockburn, A., Cunningham, W., Fowler, M., ... \& Kern, J. (2001). Manifesto for agile software development.

[14]http://www.thingiverse.com/thing:1155687

[15]Helman, P. (2016) Kanye West's Updated The Life Of Pablo Is Now On Apple Music And Spotify. Stereogum. http://www.stereogum.com/1868554/kanye-wests-updated-the-life-of-pablo-will-reportedly-be-on-apple-music-and-spotify-thisfriday/news/
} 